\title{
Revisiting the census of low-luminosity AGN
}

\author{
A. Capetti \\ INAF - Osservatorio Astronomico di Torino, via Osservatorio 20, 10025 Pino Torinese, Italy \\ e-mail: capetti@oato.inaf.it
}

Received 23 August 2011 / Accepted 20 September 2011

\section{ABSTRACT}

\begin{abstract}
The aim of this paper is to revisit critically the current census of active galactic nucleus (AGN) as derived from optical spectroscopy. We considered the spectra of nearby $(z<0.1)$ galaxies from the Sloan Digital Sky Survey (SDSS). The equivalent width (EW) distribution of the $[\mathrm{O} \mathrm{III}] \lambda 5007$ emission line is strongly clustered around $\sim 0.6 \AA$, extending the validity of the results we obtained for red giant ellipticals. The close connection between emission lines and stellar continuum points to stellar processes as the most likely source of the bulk of the ionizing photons in these galaxies although their emission line ratios are similar to those of active nuclei. Genuine AGN might be sought mainly among the minority $(\sim 5-10 \%)$ of outliers, i.e., galaxies with EW $\gtrsim 2 \AA$. The galaxies located in the AGN region of the spectroscopic diagnostic diagrams outnumber outliers by a factor 5-10, which casts doubts on the accuracy of the current identification of active galaxies, particularly those of LINERs of low line luminosity, $\lesssim 10^{39}-10^{40} \mathrm{erg} \mathrm{s}^{-1}$.

This conclusion can be tested by using spectra that cover smaller physical regions such as those that are already available in the literature of the $\sim 500$ nearest bright galaxies, with a stellar continuum reduced by a factor of $20-100$ with respect to SDSS galaxies. If the emission lines were mainly of AGN origin, their contrast against the continuum should be enhanced. Instead, their EW distribution is similar to that of the SDSS sample, with just an increase of the outlier fraction.

We conclude that the number of low-luminosity AGN is currently largely overestimated with a sample purity as low as $\sim 10 \%$. As a consequence the properties of low-luminosity AGN should be fundamentally revised.
\end{abstract}

Key words. galaxies: active - galaxies: ISM

\section{Introduction}

Emission lines are among the most widely tools used to reveal an active galactic nucleus (AGN) and to explore the properties of its central engine. The luminosity of narrow emission lines not affected by nuclear obscuration, is a robust estimator of the AGN bolometric luminosity (Mulchaey et al. 1996). Diagnostic diagrams that compare emission line ratios can distinguish H II regions from gas ionized by nuclear activity (Heckman 1980) and separate AGN into various sub-classes, e.g., Seyferts and LINERs $^{1}$ (Kewley et al. 2006), possibly associated with different accretion modes (Buttiglione et al. 2010). The analysis of extensive spectroscopic datasets can then be used to obtain a detailed census of active galaxies, to study their properties and to look for statistical connections with, e.g., the host galaxies and environment.

In an initial analysis (Capetti \& Baldi 2011, hereafter Paper I) we studied the spectra of a large sample of nearby, red, giant early-type galaxies (RGEs) from the Sloan Digital Sky Survey (SDSS). We restricted the analysis to galaxies with a $\mathrm{Ca}$ break strength $D_{n}(4000)>1.7$, with a stellar velocity dispersion $\sigma_{*}>156 \mathrm{~km} \mathrm{~s}^{-1}$ (corresponding to a mass $M_{*} \gtrsim 5 \times 10^{10} M_{\odot}$, Hyde \& Bernardi 2009) and retained only early-type galaxies, i.e., objects with a concentration index $C_{r} \geq 2.86$ (Nakamura et al. 2003; Shen et al. 2003).

Most of these galaxies show emission lines in their spectra, e.g., the [O III] $\lambda 5007$ emission line is detected in $53 \%$ of them. Furthermore, the vast majority of RGEs for which it is possible to derive emission line ratios (amounting to about half of

\footnotetext{
${ }^{1}$ Low ionization nuclear emission regions (Heckman 1980).
}

the sample) show values characteristic of LINERs. These results apparently lead to the conclusion that most of these nearby luminous galaxies harbor an active nucleus.

However, the [O III] flux shows a strong correlation with the flux measured within the 3 " SDSS fiber in the $i$-band. The $i$-band magnitude (being less affected by uncertainties in the kcorrection, absorption, and by differences in the stellar population) is a good estimator of the stellar mass within the fiber. Thus the ratio between lines and stellar mass is essentially constant (showing a dispersion of only $0.18 \mathrm{dex}$ ). Furthermore, there is no significant change of EW with redshift, despite the change in the size of the region covered by the SDSS fiber from $\sim 0.6$ to $5.5 \mathrm{kpc}$. The close connection between emission lines and stellar continuum points to stellar processes as the most likely source of the bulk of the ionizing photons in RGEs, rather than active nuclei. In particular, it has been suggested that hot evolved stars can play a dominant role (e.g., Trinchieri \& di Serego Alighieri 1991; Binette et al. 1994), an idea also supported by the results obtained from photoionization models that are able to reproduce the observed EW and optical line ratios (e.g., Stasinska et al. 2008; Sarzi et al. 2010). Shocks driven by supernovae or stellar ejecta might also contribute to the ionization budget. Conversely, these results are not straightforward to explain if the emission lines are powered by an AGN.

A minority, $4 \%$, of the galaxies show emission lines with an equivalent width a factor of $\gtrsim 2$ greater than the sample median. Seyfert-like spectra are found only among these outliers. Furthermore, $40 \%$ of this subgroup have a radio counter part, against $\sim 6 \%$ of the rest of the sample. These characteristics argue in favor of an AGN origin for their emission lines. However, 

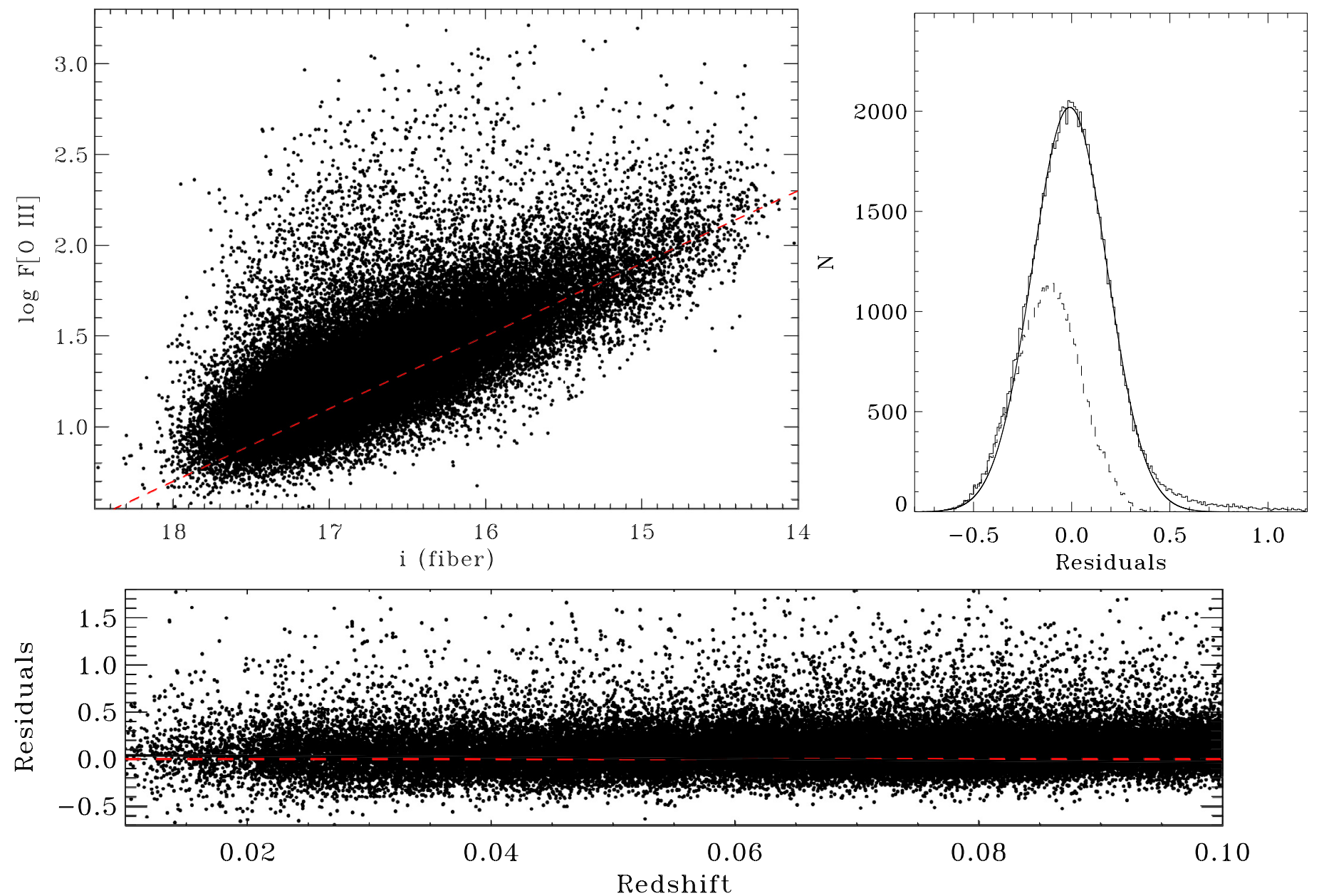

Fig. 1. Left panel: logarithm of the [O III] emission line flux (in units of $10^{-17} \mathrm{erg} \mathrm{s}^{-1} \mathrm{~cm}^{-2}$ ) versus the k-corrected $i$-band magnitude within the SDSS fiber, both quantities corrected for galactic absorption. For clarity, upper limits in the line flux are not reported. The dashed red line corresponds to a constant ratio between the two quantities. Right panel: histogram of the residuals from the median line. The dashed histogram is the contribution of upper limits. The solid line is a Gaussian distribution with a width of 0.19 dex. Bottom: residuals from the median vs. redshift.

emission line diagnostic diagrams do not reveal a distinction between the AGN subset and the other members of the sample, and consequently they are not sufficient to establish the dominant source of the ionizing photons, which is better predicted by the EW of the emission lines.

In this paper we relax the selection criteria, expanding our analysis to all nearby galaxies with spectra available from the SDSS Data Release (DR) 7. While in Paper I we focused only on giant elliptical galaxies, in Sect. 2 we consider all spectra with a Ca break strength $D_{n}(4000)>1.7$, i.e., all galaxies for which the SDSS fiber covers an old stellar population, dropping the requirements on mass and morphology. In Sect. 3 we move to the analysis of all SDSS DR7 spectra of nearby galaxies, regardless of their spectro-photometric properties. In order to improve the AGN census to the lowest luminosities, in Sect. 4 we also consider the 486 nearby galaxies studied spectroscopically by Ho et al. (1995) with the Hale telescope at Mount Palomar.

We discuss our results in Sect. 5 and present our summary and conclusions in Sect. 6.

\section{Emission lines properties of red galaxies}

We here consider the $\sim 100000$ SDSS (York et al. 2000) Data Release (DR) 7 spectra characterized by a Ca break strength
$D_{n}(4000)>1.7$ (and an error $\left.<0.05\right)^{2}$, associated with $\sim 90000$ unique galaxies at $z<0.1$ based on the MPA-JHU DR7 spectrum measurements, available at http: //www . mpa-garching . mpg.de/SDSS/DR7/. Then, we are studying all nearby galaxies for which the SDSS fiber covers an old stellar population. For the sake of brevity we define them as "red galaxies". Most of them are early-type galaxies, now spanning their full range of mass, but there is also a substantial number of spiral galaxies where the bulge is sufficiently extended to fill the SDSS fiber.

\subsection{Emission lines and stellar continuum}

We considered the [O III $] \lambda 5007$ emission line flux measured after subtraction of a starlight template (see Kauffmann et al. $2003 \mathrm{~b}$ for a detailed description of the method used for the continuum subtraction). The [O III] line is detected at a significance higher than $3 \sigma$ in $\sim 53 \%$ of the red galaxies. Figure 1 compares the [O III] flux with the k-corrected $i$-band magnitude within the SDSS fiber, both quantities corrected for galactic absorption. The strong connection found in Paper I is confirmed with the vast majority of the red galaxies clustered in a narrow stripe.

2 This corresponds effectively to the inclusion only of spectra with a median signal-to-noise ratio higher than $\sim 10$. 


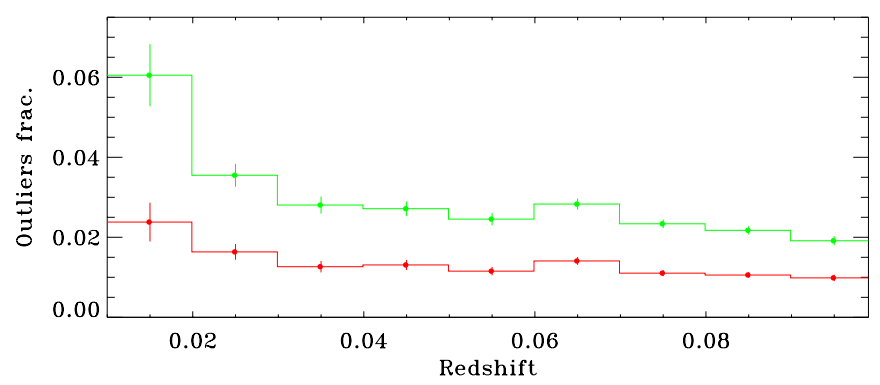

Fig. 2. Fraction of outliers versus redshift for the red galaxies, dominated by an old stellar population, i.e., with $D_{n}(4000)>1.7$. Green dots and histogram are weak outliers, the red ones are strong outliers.

Slope and intercept of the best-fitting logarithmic linear relation are indistinguishable from those derived for RGEs.

In the right panel we show the histogram of the residuals from the median line (the dashed histogram is the contribution of upper limits) that is well reproduced by a Gaussian distribution with a dispersion of only 0.19 dex (similar results, although with slightly broader distributions, are obtained using different lines and/or continuum bands). Nonetheless, there are outliers from this trend. Setting a threshold at $\mathrm{R}[\mathrm{O} \mathrm{IIII}]^{3}=5$ we find $1.2 \%$ of "strong" outliers, a fraction that increases to $2.5 \%$ of "weak" outliers when lowering the limit to $3^{4}$.

A more readily measurable parameter is the $\mathrm{EW}$ of the [O III] line. For the SDSS galaxies it has been estimated by considering the continuum level 200 pixels around the line. The EW distribution is also highly concentrated with a median value of $\sim 0.6 \AA$ (including also upper limits in the analysis) and a dispersion of 0.21 dex.

We looked for trends between the residuals and the spectrophotometric parameters used for the sample selection (namely redshift and $D_{n}(4000)$ ) but we failed to find any statistically significant link. There is instead a weak trend for an increase of the fraction of outliers moving from higher to lower redshifts (see Fig. 2) particularly clear in the two bins at the lower redshifts.

As we already discussed in Paper I, because we are studying emission lines that are generally of very low EW, it is important to assess the reliability of these measurements and of the error estimates. The comparison of the results obtained from duplicated observations of the same galaxies indicates that the assessment of the statistical errors provided by the SDSS database is robust. Indeed the differences between pairs of measurements closely match those predicted by their uncertainties. The good agreement of the flux measurements obtained by Oh et al. (2011) using a different approach for the removal of the stellar emission also supports the robustness of those provided by MPA-JHU database.

We also considered the possibility of an additional error related to the accuracy of the subtraction of the stellar emission (see Annibali et al. 2010) by adding in quadrature a constant error in EW of $0.16 \AA$ for $\mathrm{H} \beta$ and of $0.08 \AA$ for the other key emission lines. Our results on the connection between [O III] flux and $z$ magnitude are only marginally changed by adopting this more conservative error treatment. The fraction of detected sources decreases from 53 to $49 \%$, but the slope of the relation remains unchanged. The number of outliers is not affected. We anticipate that, similarly, the results on the spectroscopic diagnostic

\footnotetext{
${ }^{3} \mathrm{R}[\mathrm{O} \mathrm{III}]$ is defined as the ratio between the [O III] flux of a galaxy with respect to its median value at a given magnitude.

${ }^{4}$ As a comparison, the fraction of outliers for RGEs is $0.5 \%$ for $\mathrm{R}[\mathrm{O} \mathrm{III}]>5$ and $1.2 \%$ at $\mathrm{R}[\mathrm{O} \mathrm{III}]>3$.
}

diagrams, presented in the next section, are not substantially affected; the main difference is a reduced fraction of galaxies that can be classified, but their general location is not changed.

\subsection{Spectroscopic diagnostic diagrams}

Figure 3 shows the location in the spectroscopic diagnostic diagrams (e.g., Heckman 1980; Baldwin et al. 1981; Veilleux \& Osterbrock 1987; Kewley et al. 2006) for the selected galaxies that have all relevant emission lines detected at $S N R>3$ separately for each diagram. Starting from the left side, the percentages with respect to the whole sample are 30,25 , and $8 \%$ in the three diagrams, respectively. The majority of the objects fall in the LINERs region, while the Seyfert and star-forming regions are scarcely populated. In particular (according to their location in the left diagram) only $0.6 \%$ of the sample have line ratios typical of star-forming galaxies, $10.8 \%$ are the composite galaxies, while $18.9 \%$ of the red galaxies are in the AGN region.

With respect to the overall population, our selection filters out most of the objects at (or close to) the location of starforming galaxies. This can be explained as the consequence of the exclusion of the objects with a substantial population of young stars whose emission lines have a significant contamination from star-forming regions.

Approximately $\sim 70 \%$ of the galaxies have no optical classification because at least one of the key emission lines is undetected. Nonetheless, in $85 \%$ of them, the [N II] and $\mathrm{H} \alpha$ lines are both detected; this allows us to derive at least a crude classification. Indeed, $\log ([\mathrm{N} \mathrm{II}] / \mathrm{H} \alpha)>-0.3$ in $70 \%$ of them (see Fig. 4 ), a threshold above which no star-forming galaxies are found. On the other hand, Seyfert galaxies have usually bright emission lines, therefore they are expected to be all properly cataloged by the diagnostic diagrams in Fig. 3. This leads to the conclusion that these galaxies, which amount to more than half of the sample, generally are LINERs.

Let us now consider the properties of outliers, i.e., the objects with EW exceeding by a factor of at least 3 the median of the sample. "Strong" outliers (i.e., the objects with R[O III] $>5$ ) essentially represent the totality of Seyferts, but many of them are LINERs, while weaker outliers (with $3<\mathrm{R}[\mathrm{O} \mathrm{III}]<5$ ) are mostly LINERs. It is important to note that they cannot be readily separated from the bulk of the red galaxies population.

We conclude that the main results obtained in Paper I are confirmed by the analysis of all "red galaxies", regardless of their mass, suggesting that the bulk of red emission line galaxies are not genuine AGN. While $\sim 20 \%$ of the red galaxies are located in the AGN region of the diagnostic diagrams (and the fraction of LINERs is probably as high as $\sim 70 \%$ based on the $[\mathrm{N} \mathrm{II}] / \mathrm{H} \alpha$ ratio), the majority of them have emission lines most likely powered by stellar processes. Genuine AGN might be sought mainly among the $\sim 1-2 \%$ of outliers, i.e., galaxies with higher EW values. The number of AGN hosted by red galaxies derived from the emission line ratios is probably overestimated by a factor ranging from 10 up to 70 .

\section{Emission line properties of nearby galaxies}

We now also drop the requirement of a large Ca break, but, for consistency, we maintain the requirement that its error must be smaller than $<0.05$ and the restriction on redshift $(z<0.1)$. This selection yields $\sim 315000$ spectra.

Because we are mainly interested in the study of AGN we excluded from the analysis the $\sim 155000$ galaxies spectra with 


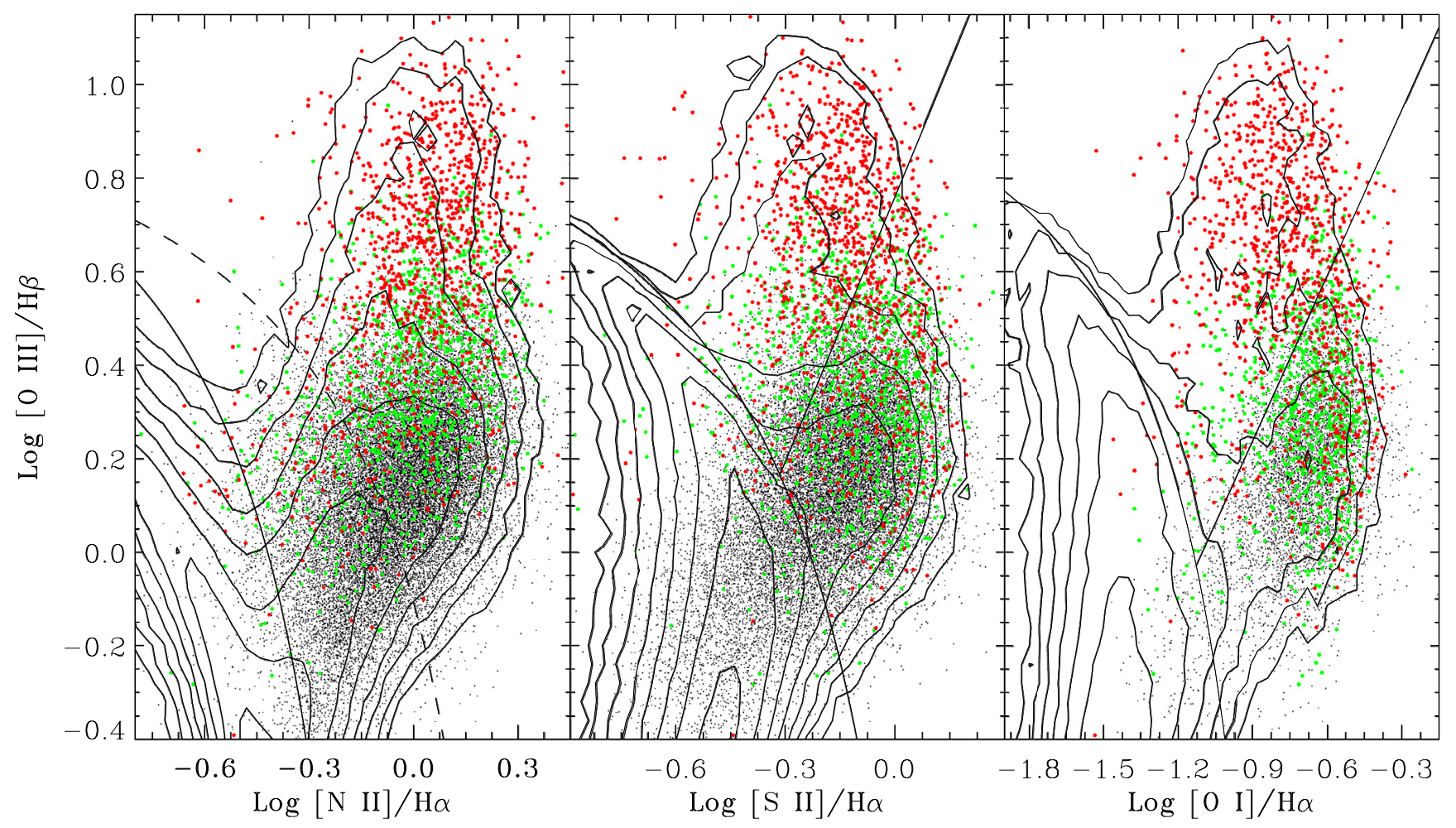

Fig. 3. Spectroscopic diagnostic diagrams for the red galaxies. The solid lines are from Kewley et al. (2006) and separate star forming galaxies, LINER, and Seyfert; in the first panel the region between the two curves is populated by the composite galaxies. Small black dots mark the location of all red galaxies, while the large colored dots mark the outliers, i.e., objects with a line excess with respect to the median value by a factor of $\mathrm{R}[\mathrm{O} \mathrm{III}]>5$ (red) or 3 (green) in Fig. 1. Contours represent the iso-densities of all DR7 emission line galaxies with $z<0.1$.

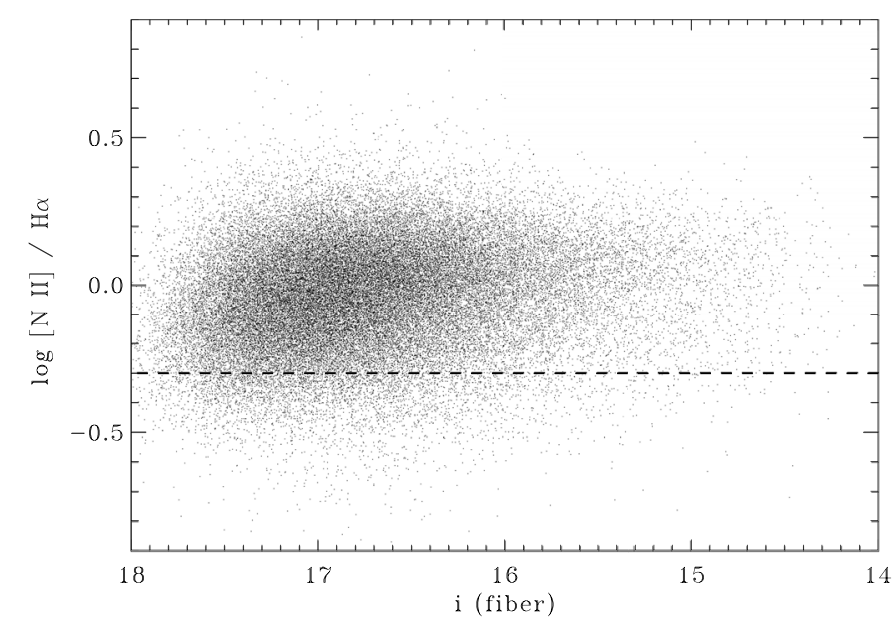

Fig. 4. $[\mathrm{N} \mathrm{II}] / \mathrm{H} \alpha$ ratio for all red galaxies with both lines detected. Emission lines with a ratio that locate an object above the horizontal dashed line are unlikely to be powered by star formation.

emission line ratios that locate them in the diagnostic diagram in the region characteristic of star forming and, at least in a first stage, also the $\sim 39000$ composite galaxies.

The [O III] line is detected in $46 \%$ of the remaining $\sim 121000$ spectra and we recovered the strong connection between line flux and stellar continuum. The distribution of the residuals from the median line (the same derived in Sect. 2.1) is still highly concentrated, see Fig. 5. The peak of the distribution is well described by a Gaussian with a width of 0.20 dex and shifted with respect to that reproduced in Fig. 1 by only 0.01 dex. However, there is a more prominent tail of galaxies with higher

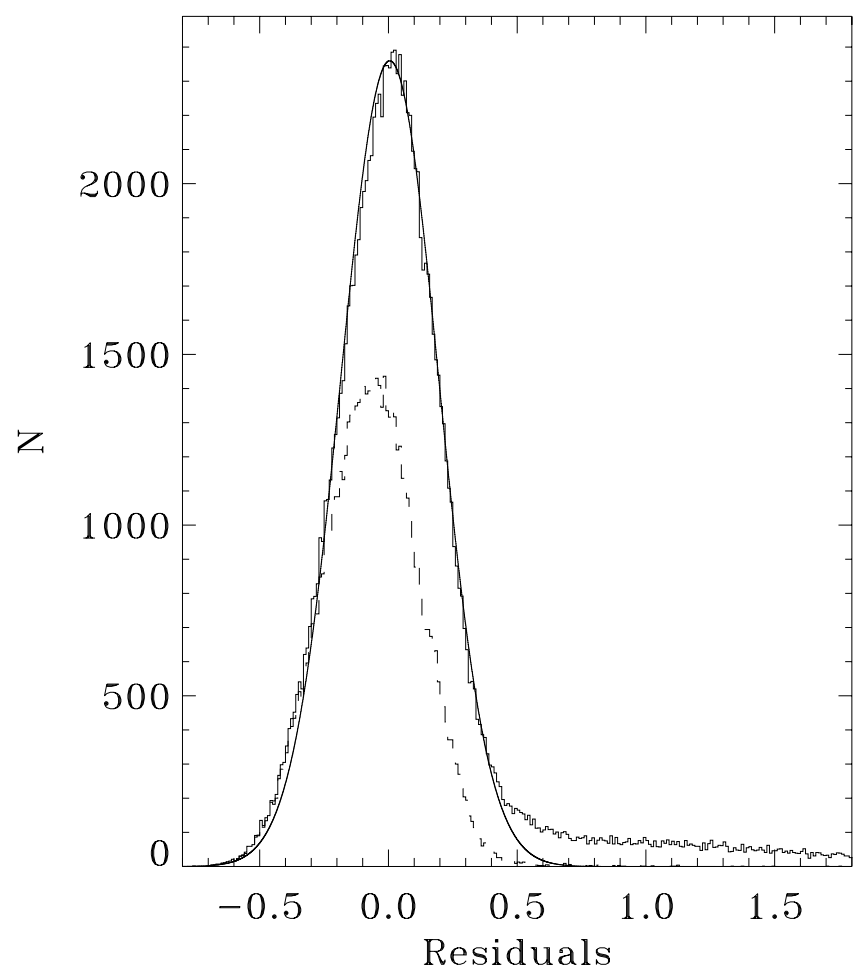

Fig. 5. Distribution of the residuals from the median line for all nearby DR7 galaxies. The dashed histogram is the contribution of upper limits. The solid line represents a Gaussian distribution with a width of 0.20 dex.

ratios, with $5.8 \%$ of outliers with $\mathrm{R}[\mathrm{O}$ III] $>5$ (7.6\% for R[O III] $>3$ ), i.e., 3-5 times more than for the red galaxies. 


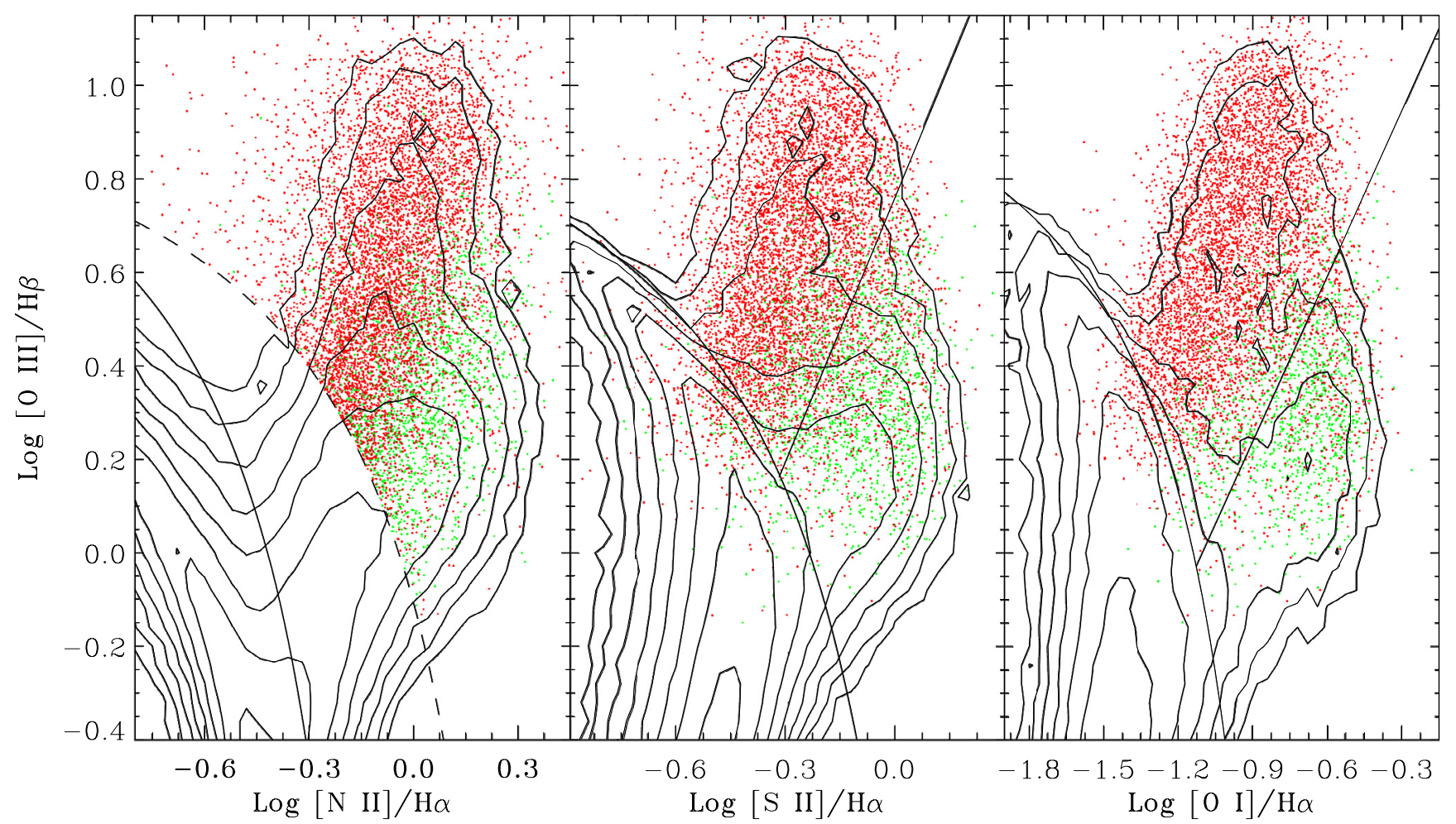

Fig. 6. Spectroscopic diagnostic diagrams for all DR7 emission line galaxies with $z<0.1$. Contours represent their iso-densities and are drawn with a common ratio of 2 . Dots show the location of the outliers (i.e., objects with a line excess with respect to the median value by a factor of $\mathrm{R}[\mathrm{O} \mathrm{III}]>5$ (red) or 3 (green) in Fig. 1) limiting to those falling in the AGN in the left diagram, i.e., excluding star-forming and composite galaxies.

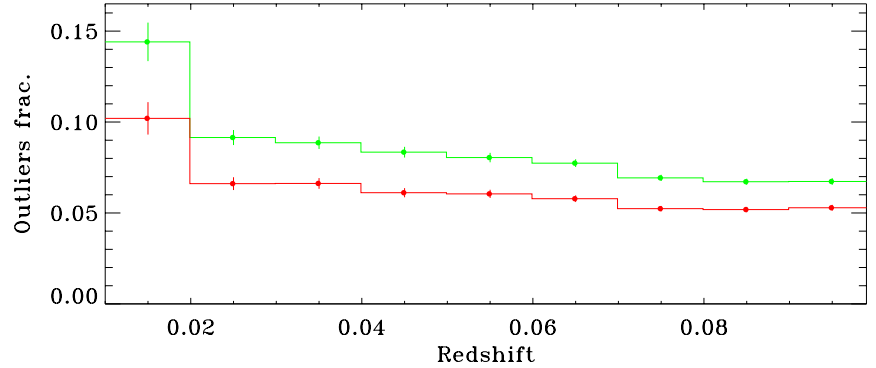

Fig. 7. Fraction of outliers versus redshift for all galaxies with $z<0.1$ and located in the AGN region in the left panel of Fig. 6. The weak histogram is for weak outliers, the red one for strong outliers.

There is again a weak increase of the fraction of outliers from higher to lower redshifts (see Fig. 7), but less pronounced than that observed for the red galaxies. Here there are $\sim 7 \%$ outliers at $z \sim 0.1$, increasing to $\sim 8 \%$ for $z \sim 0.02-0.03$, and only in the first bin $(z \sim 0.01-0.02)$ the fraction reaches $\sim 14 \%$.

In the diagnostic diagrams we can locate $\sim 24 \%$ of these galaxies. Because we excluded all star-forming and composite galaxies, these all lie in the AGN region in Fig. 6. Strong outliers are mostly located in the Seyfert region, while the weak ones are generally LINERs. Furthermore, in $65 \%$ of them we measure $\log ([\mathrm{N} \mathrm{II}] / \mathrm{H} \alpha)>-0.3$, which argues in favor of a LINER classification.

The selection of outliers based on their higher EW also induces a separation between them and the rest of the galaxies of the sample in terms of line luminosity, $\left.L_{[\mathrm{O}} \mathrm{III}\right]$. Figure 8 shows the distributions of $L_{[\mathrm{O} \text { III] }}$ of the sources located in the AGN region in Fig. 6 separately for the galaxies of low EW and for the outliers. Strong outliers have a median luminosity of $10^{40} \mathrm{erg} \mathrm{s}^{-1}$, $\sim 6$ times higher than the rest of the sample. If we refer $L_{[\mathrm{O} \text { III] }}$ to the Eddington luminosity (adopting the Tremaine et al. 2002 relation between black hole mass and stellar velocity dispersion) the ratio between the two medians increases to a factor of $\sim 20$.

If we adopt a less conservative AGN definition, i.e., not excluding the composite galaxies, the objects with a [O III] detection are $59 \%$. The results in terms of the distribution of the residuals are similar, but with a slightly higher fraction of outliers $(7.6 \%$ and $11.5 \%$ at $\mathrm{R}[\mathrm{O}$ III] $>3$ and 5, respectively); those that can be located in a least one of the diagnostic diagrams are instead $42 \%$, and $67 \%$ of them have $\log ([\mathrm{N} \mathrm{III}] / \mathrm{H} \alpha)>-0.3$.

Therefore, again, although the fraction of outliers is significantly higher than for the "red galaxies", most of the nearby galaxies are likely to have emission lines powered by stellar processes. The ratio between objects with line ratios typical of AGN and outliers (the likely genuine AGN) is still very high, a factor of $\sim 5-10$.

\section{Revisiting the Palomar survey}

Because the dominant contamination to the emission lines is apparently associated with stellar processes, significant progress for a proper AGN census can be obtained considering spectra obtained in a smaller physical region, with a reduced contribution from the stellar emission. These data are already available in the literature from the spectroscopic survey performed at the Palomar observatory of 486 nearby (with an average recession velocity of $\sim 1000 \mathrm{~km} \mathrm{~s}^{-1}$ ) bright galaxies (Filippenko \& Sargent 1985). The objects of the "Palomar sample" have a median distance 20 times smaller than the SDSS galaxies and as consequence the emission associated with AGN of lower luminosity may be visible in their spectra. 

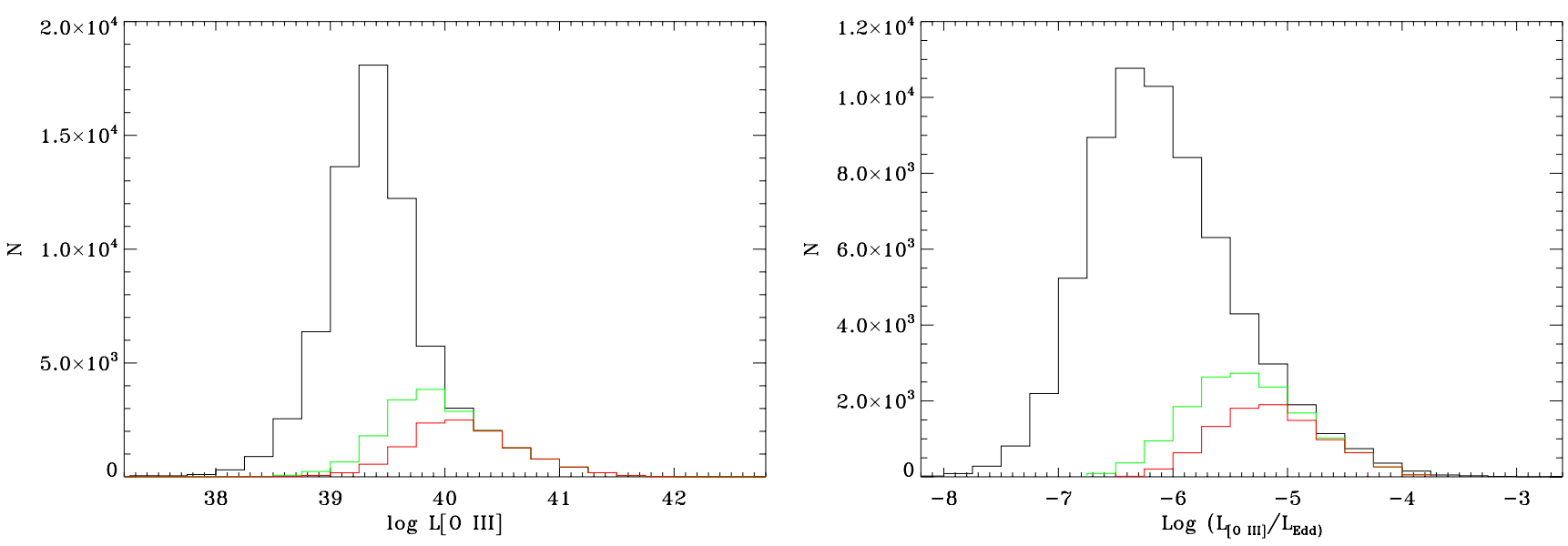

Fig. 8. Left: histogram of the [O III] luminosity (in $\mathrm{erg} \mathrm{s}^{-1}$ ) for all galaxies located in the AGN region in Fig. 6; the contribution of strong (weak) outliers is shown in red (green). Right: same as the left panel, but with the [O III] luminosity measured in fraction of the Eddington luminosity.

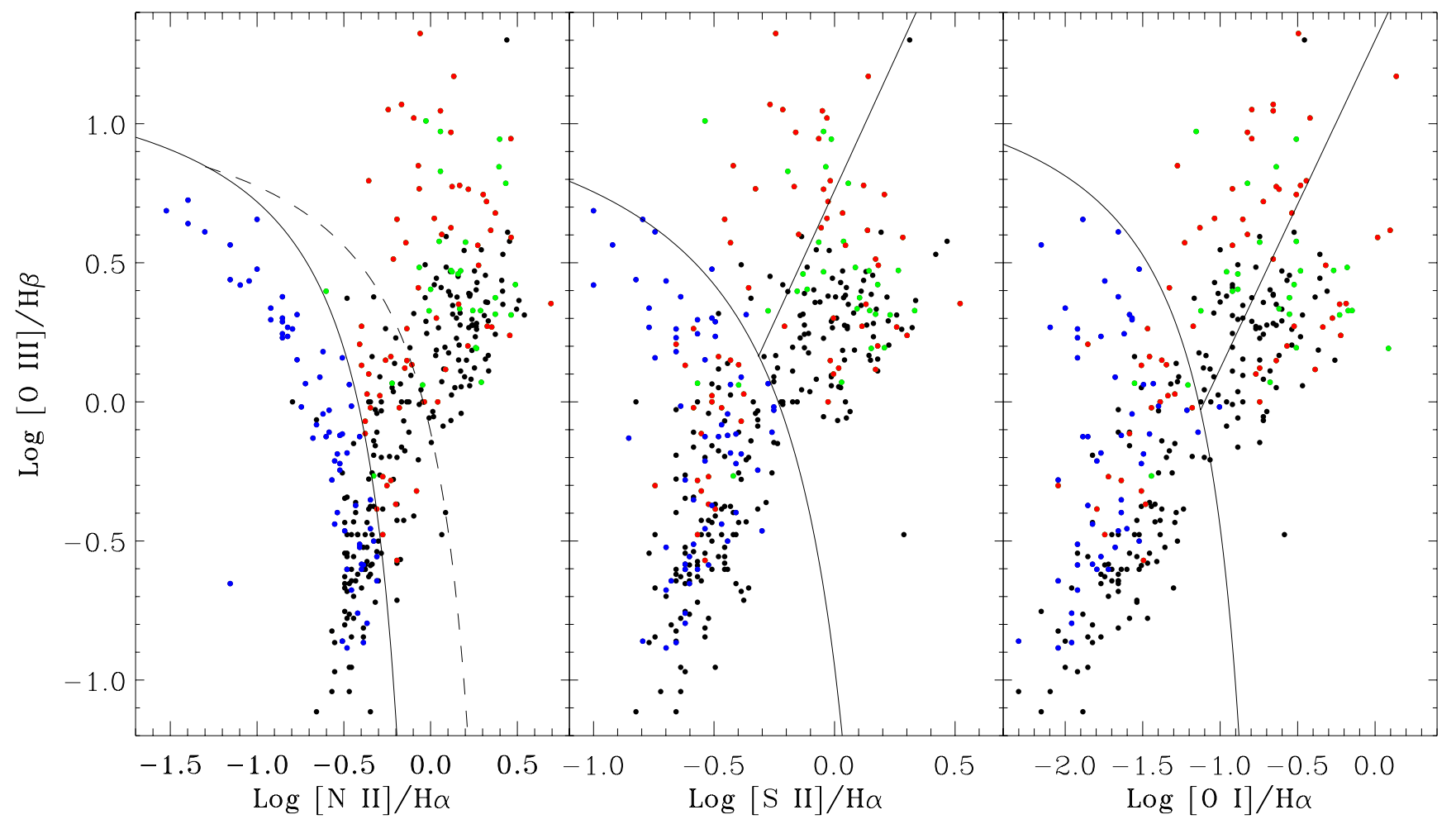

Fig. 9. Spectroscopic diagnostic diagrams for the Palomar sample, adapted from Ho et al. (1997). Black dots mark the location of all galaxies of low EW (see text for details). Colored dots mark the outliers, i.e., objects with a line excess with respect to the median value measured for "red galaxies" by a factor of R[O III] $>5$ (red) or 3 (green). Blue dots are the star-forming galaxies according to their location in the left panel.

Optical spectra were obtained at the Hale $5 \mathrm{~m}$ telescope at Palomar Observatory for almost all bright galaxies in the northern sky, selected from the Revised Shapley-Ames Catalog of Bright Galaxies (Sandage \& Tammann 1981) with a limit of $\mathrm{B}_{\mathrm{T}}<12.5$ (Ho et al. 1995, 1997). A $2^{\prime \prime}$ slit was generally used and the spectra were extracted over a synthetic aperture of $4^{\prime \prime} \times 22^{\prime \prime}$. Coincidentally, this extraction region has the same size as the circular, $3^{\prime \prime}$ in radius, fiber of the SDSS.

Only 68 galaxies turn out be lineless, while 418 objects are emission line galaxies ${ }^{5}$. It is possible to locate a very high part of

\footnotetext{
5 From this initial list we excluded a few sources not formally part of the sample and included for historical reasons, mainly AGN with a negative declination.
}

these galaxies in the diagnostic diagrams (see Fig. 9), between 66 and $76 \%$, depending on the pair of ratios considered. Based on their position in the left diagram, $38 \%$ of them are AGN, $15 \%$ are composite galaxies, and $24 \%$ are star-forming galaxies ${ }^{6}$.

Ho et al. (1997) have listed the EW values for the $\mathrm{H} \alpha$ line; we used these data to measure the ratio between the [O III] and the continuum around the $\mathrm{H} \alpha$ line. Although this is not a conventional measurement, we can estimate this same parameter for the red galaxies discussed in Sect. 2.1 for reference.

The distribution of $\mathrm{EW}_{[\mathrm{O} \mathrm{III}] @ \mathrm{H} \alpha}=\log F_{[\mathrm{O} \text { III }]} / F_{\text {cont, } \mathrm{H}_{\alpha}}$ of the SDSS red galaxies is well reproduced by a Gaussian centered at

${ }_{6}$ The remaining galaxies are $14 \%$ lineless and $9 \%$ that have at least one of the 4 relevant emission lines undetected. 


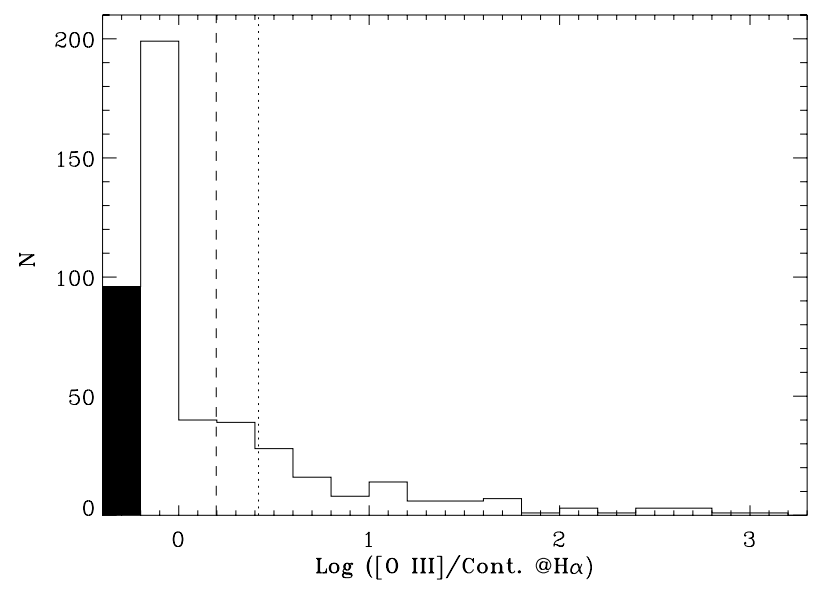

Fig. 10. Logarithm of the ratio between the [O III] flux and the continuum around the $\mathrm{H} \alpha$ line for the Palomar sample. The vertical lines are located at 3 and 5 times the median value measured for the SDSS red galaxies, i.e., $0.52 \AA$. The first bin of the distribution contains the 28 emission line galaxies where the [O III] line is not detected and the 68 lineless galaxies.

$0.52 \AA$. The distribution of $\mathrm{EW}_{[\mathrm{O} \mathrm{III}] @ \mathrm{H} \alpha}$ for the Palomar sample is shown in Fig. 10. The first two bins (including galaxies with values $<1 \AA$, the 28 emission line galaxies where the [O III] line is not detected and the 68 lineless galaxies) contain more than $60 \%$ of the sample. Although the statistics is more limited than for the SDSS galaxies, the emission line galaxies in the Palomar sample are also mainly objects of low equivalent width.

In particular, only $1 / 3$ of the 175 galaxies located in the AGN region of the diagnostic diagrams have $\mathrm{EW}_{[\mathrm{O} \mathrm{III}] @ \mathrm{H} \alpha}>$ $3 \times 0.52 \AA$, reducing the fraction of likely active nuclei from $38 \%$ to $12 \%$ (and there are only $5 \%$ of composite galaxies). Moving the threshold to $5 \sigma$, there are $7 \%$ AGN and $4 \%$ composites.

The results obtained from the diagnostic diagrams built considering $[\mathrm{S} \mathrm{II}] / \mathrm{H} \alpha$ and $[\mathrm{O} \mathrm{I}] / \mathrm{H} \alpha$ ratios are similar: the percentage of AGN decreases from $\sim 32-35 \%$ to $\sim 12 \%$ (and $8 \%$ at $5 \sigma$ ). This is mostly owing to a strongly reduced number of LINERs, because less than $20 \%$ of them have an equivalent width higher than the adopted threshold. In contrast, only a minority of (possible) Seyferts have a low EW and they are generally located close to the boundary with LINERs.

The line luminosity of the Palomar galaxies located in the AGN region is a factor of $\sim 10$ lower than the SDSS galaxies, reaching values as low as $\left.L_{[\mathrm{O}} \mathrm{III}\right] \sim 10^{37} \mathrm{erg} \mathrm{s}^{-1}$. The outliers form the bright end of this distribution and have a median of $\left.L_{[\mathrm{O}} \mathrm{III}\right] \sim 10^{39} \mathrm{erg} \mathrm{s}^{-1}$.

\section{Discussion}

The spectra of nearby $(z<0.1)$ galaxies extracted from the SDSS DR7 show that even after removing the star-forming galaxies, most of the galaxies are emission line galaxies. The percentage of objects falling into the AGN region of the diagnostic diagrams is $\sim 24 \%$, generally showing line ratios typical of the subcategory of LINERs. Considering the objects for which an identification can be obtained from the $[\mathrm{N} \mathrm{II}] / \mathrm{H} \alpha$ ratio as well, this percentage is increased to $\sim 65 \%$. Apparently, this is an indication that most of these galaxies host an active nucleus.

However, their distribution of line equivalent widths is strongly clustered around $\left.\mathrm{EW}_{[\mathrm{O}} \mathrm{III}\right] \sim 0.6 \AA$. This result is very difficult to account for if the emission lines are powered by an AGN because this requires a fine-tuning between the strength of the nuclear ionizing field, the spatial distribution of the emission lines, and the stellar mass. Conversely, the strong connection between line emission and stellar continuum points to a stellar origin of the emission lines. As already noted in the introduction, the observed EW and optical line ratios are consistent with the predictions of photoionization from hot evolved stars. Only the minority of galaxies $(\lesssim 10 \%$ of the sample) that does not obey this trend, because they have higher EW, should be considered as genuine AGN.

An analogy can be drawn between the origin of emission lines and the source of dust heating: recent Herschel observations of M 81 and M 33 (Bendo et al. 2010; Boquien et al. 2011) show that the diffuse photon field produced by the evolved stellar population plays a fundamental role in setting the dust temperature, competing against young stars and (in the case of M 81) the active nucleus in setting the energy budget.

A test to probe the dominant role of old stars can be performed considering objects located at different distances by only assuming that the galaxy's population does not vary with redshift (a point that we will consider in more detail below). If the emission lines were of nuclear origin, their contrast against the stellar continuum should be enhanced in closer objects, leading to an increase of the lines EW, because the fixed angular size of the fiber covers a smaller portion of the galaxy. Conversely, in case of a dominant stellar origin, lines are produced over an extended region and no significant changes are expected in the distribution of equivalent width with redshift. This is the result of our analysis, because the peak of the distribution of the ratio between line flux and continuum does not vary significantly with redshift.

The Palomar survey provides us with the data required to perform an even more stringent test because it includes objects at an average distance $\sim 20$ times smaller than the SDSS galaxies (their average redshift is $z=0.063$ ). Although the average surface brightness within the extraction region for the Palomar sources is $\sim 2-3$ mag higher than for the SDSS sample, this only partly compensates for the strongly reduced physical size of the extraction region, which leads to a typical decrease of the stellar continuum by a factor of $20-100$. However, the distribution of the ratio between line and continuum still shows a very strong concentration at low values, with more than $60 \%$ objects having values $<1 \AA$, confirming that the change in the stellar continuum is closely coupled with a decrease in the line flux. This again points toward the conclusion that most of the line emission originate from an extended region.

Nonetheless, a change with redshift is observed in the fraction of outliers that is slightly higher in closer SDSS galaxies, increasing from an average of $7.6 \%$ to $14 \%$ for the objects with $z \sim 0.01-0.02$. This result can be reconciled with the stable location of the peak of the EW distribution if, in addition to the dominant diffuse line emission, there is also a contribution from a nuclear emission line region. In the more distant objects, this is generally swamped by the stellar emission. This might be sufficient to increase the lines EW within the aperture in nearer galaxies at the same level of luminosity and give rise to an outlier. This is confirmed by a further increase of likely active galaxies among the Palomar galaxies, where one third of the objects in the AGN region also show a high EW, twice the fraction of the nearer SDSS galaxies.

The distribution of the [O III] luminosity of the outliers indicates that we can currently isolate AGN in SDSS galaxies down to $\left.L_{[\mathrm{O}} \mathrm{III}\right] \sim 10^{39} \mathrm{erg} \mathrm{s}^{-1}$, but most of them are 10 100 times brighter. This limit decreases by a factor of $\sim 10$ for 
a few Palomar galaxies, but even in this sample the bulk of the AGN have $L_{[\mathrm{O} \text { III] }} \gtrsim 10^{39} \mathrm{erg} \mathrm{s}^{-1}$.

In principle, the information on the variation of the fraction of likely AGN with redshift could be used to statistically model the luminosity function of lower-luminosity AGN. However, as noticed above, the comparison between the galaxies from the SDSS and Palomar surveys (but even with the same survey but at the different redshift) should be treated with some care. Indeed, the selection criteria of the two surveys are different and, within the SDSS sample, the requirements to include a galaxy in the spectroscopic database (Strauss et al. 2002) causes a change of the target properties with redshift. If there is a relation between the line emission and host properties, particularly in case of an AGN origin, this might have an impact on the fraction of outliers at various redshift. For example, several local low-mass galaxies are included in the Palomar sample that would not enter in the SDSS spectroscopic dataset were they located at the median SDSS redshift. While the SDSS galaxies have an absolute magnitude mostly in the range $M_{\mathrm{r}}=-20-23$, in the Palomar sample $\sim 15 \%$ of the galaxies do not fall into this range, because they have a lower luminosity. This low fraction does not alter our general result significantly, even in the assumption that i.e., low-mass galaxies do not host active nuclei, but a more detailed comparison requires a careful matching of the samples.

Our results point to the conclusion that the census of AGN based on the optical spectroscopic diagnostic diagrams currently includes a dominant percentage of objects (possibly as high as $90 \%$ ) where the emission lines are not powered by an active nucleus. This is particularly severe for the lowest luminosity AGN, which remain virtually unexplored with the currently available data. This clearly has strong implications for our understanding of the origin and evolution of AGN. Indeed, a statistical study based on the analysis of sample whose purity is only at the $\sim 10 \%$ level necessarily leads to flawed results. The situation is slightly better for the nearer Palomar galaxies, but in this sample as well only one third of the objects usually classified as AGN are likely to be genuine active galaxies.

As a consequence the properties of low-luminosity AGN, e.g., their connection with the host galaxies and environment, should be fundamentally revised. This problem is particularly severe for objects of low line luminosity $\left(\lesssim 10^{39}-10^{40} \mathrm{erg} \mathrm{s}^{-1}\right)$ with line ratios typical of LINERs.

\section{Summary and conclusions}

In Paper I we showed that most nearby $(z<0.1)$, red giant earlytype galaxies are emission line galaxies and generally show emission line ratios characteristic of LINERs. Apparently, this is an indication of a very large percentage of AGN among these sources.

However, their [O III] flux shows a strong correlation with the flux measured within the $3^{\prime \prime}$ SDSS fiber in the $i$-band, a robust estimator of the stellar mass covered by the fiber used to obtain the spectra. This result is very difficult to explain if the emission lines are powered by an AGN. Conversely, it suggests that at the origin of the observed emission lines there are processes related to the stellar population. For example, the observed EW and optical line ratios are consistent with the predictions of models in which the photoionization is caused by hot evolved stars. As a consequence, only a minority of these emission line galaxies is likely to be powered by an active nucleus and this requires to revisit the current census of AGN, particularly for those of lower luminosity.
We here extended our analysis to all nearby $(z<0.1)$ SDSS galaxies. Initially we considered all galaxies where the fiber covers an old stellar population characterized by a calcium break strength greater than $D_{n}(4000)>1.7$, but removing any other constraint based on morphology or mass. As a consequence we included in the analysis early-type galaxies of any mass, as well as spiral galaxies (provided that their bulges are sufficiently extended to fill the fiber). The results obtained are effectively indistinguishable from those we found limiting our search to RGEs. The strong correlation between emission line fluxes and stellar continuum is confirmed with the same slope and intercept (within $0.03 \AA$ ). The distribution of the residuals from the median value is again found to show a dispersion of only 0.19 dex. The number of outliers, i.e., of the objects with line flux higher by a factor of at least 3 with respect to the median of the sample, is slightly higher. We found $1.2 \%$ galaxies with an line excess higher than 5 (against $0.8 \%$ for RGEs), and $2.5 \%$ for an excess of a factor of 3 (instead of $2.0 \%$ for RGEs).

By using these results as reference we explored the properties of the $\sim 300000$ galaxies in the SDSS/DR7 main sample, also dropping the constraint of the stellar population age. Excluding the star-forming galaxies, the distribution of EW turns out to be still strongly concentrated around the values found for the old stellar population galaxies, but the fraction of outliers is significantly higher $(5.1 \%$ with residuals higher than a factor of 5 and $6.6 \%$ higher than 3 ). This is probably the result of the known connection between star formation and nuclear activity (e.g., Kauffmann et al. 2003a): the selection of regions dominated by an old stellar population filters out a large part of AGN.

These results support the conclusion that stellar processes are generally at the origin of the emission lines, while active galaxies are only a small fraction of the overall galaxies population. Furthermore, the number of galaxies with AGN-like emission line ratios is far higher (by a factor of $\sim 5-10$ ) than the fraction of outliers. The location of a galaxy in the diagnostic diagrams is not a sufficient tool to establish the dominant source of the ionizing photons, which is instead better predicted by the EW of the emission lines, which in turn agrees with the conclusions of Cid Fernandes et al. (2011).

Because the dominant contamination of the emission lines is apparently associated with stars, a robust test for the reliability of the AGN census can be performed considering spectra obtained on a smaller physical region. If the emission lines are indeed mainly of nuclear origin, their contrast against the continuum is expected to be enhanced, while for a dominant stellar origin no significant changes are expected in terms of distribution of equivalent width. And this latter is the result of our analysis, because the peak of distribution of the ratio between line flux and continuum does not vary significantly with redshift. A mild increase of the fraction of outliers is instead observed at decreasing redshift. This suggests that in addition to the dominant diffuse line emission, there is also a contribution from a nuclear emission line region. In the more distant objects, this is generally swamped by the stellar emission, while in nearer galaxies the presence of the AGN is sufficient to increase the lines EW within the aperture significantly.

Even better data for this analysis come from the Palomar survey that covers most bright nearby galaxies (Filippenko \& Sargent 1985). The contribution of the stellar emission in these spectra is reduced, with respect to the SDSS sample, by a factor of 20-100. Nonetheless, the distribution of the ratio between line and continuum is still strongly concentrated at low values. The Palomar survey also confirms the trend of an AGN increase in nearer galaxies, because one third of the objects in the AGN 
region also show a high $\mathrm{EW}$, twice the fraction of the SDSS galaxies with $z \sim 0.01-0.02$.

We conclude that the current AGN census based on optical spectroscopy includes a dominant fraction of objects where the emission lines are not powered by an active nucleus, a problem particularly severe for the lowest luminosity objects with a LINER spectrum. This has strong implications for our understanding of AGN because a study based on a sample whose purity is only at the $\sim 10 \%$ level necessarily leads to spurious results. As a consequence the properties of low-luminosity AGN should be fundamentally revised.

An improved census can be obtained from the spatially resolved optical spectroscopy of the Palomar sample with a reduced aperture size, ideally with integral field units of high spatial resolution. An improvement by a factor of $\sim 10$ from the 8 squared arcseconds of the original data is easily within reach.

Acknowledgements. I thank Ranieri Baldi, David J. Axon, Roberto Rampazzo, and Francesca Annibali for their comments and useful discussions.

\section{References}

Annibali, F., Bressan, A., Rampazzo, R., et al. 2010, A\&A, 519, A40 Baldwin, J. A., Phillips, M. M., \& Terlevich, R. 1981, PASP, 93, 5 Bendo, G. J., Wilson, C. D., Pohlen, M., et al. 2010, A\&A, 518, L65
Binette, L., Magris, C. G., Stasińska, G., \& Bruzual, A. G. 1994, A\&A, 292, 13 Boquien, M., Calzetti, D., Combes, F., et al. 2011, AJ, 142, 111

Buttiglione, S., Capetti, A., Celotti, A., et al. 2010, A\&A, 509, A6

Capetti, A., \& Baldi, R. D. 2011, A\&A, 529, A126 (Paper I)

Cid Fernandes, R., Stasińska, G., Mateus, A., \& Vale Asari, N. 2011, MNRAS, 413, 1687

Filippenko, A. V., \& Sargent, W. L. W. 1985, ApJS, 57, 503

Heckman, T. M. 1980, A\&A, 87, 152

Ho, L. C., Filippenko, A. V., \& Sargent, W. L. 1995, ApJS, 98, 477

Ho, L. C., Filippenko, A. V., \& Sargent, W. L. W. 1997, ApJS, 112, 315

Hyde, J. B., \& Bernardi, M. 2009, MNRAS, 394, 1978

Kauffmann, G., Heckman, T. M., Tremonti, C., et al. 2003a, MNRAS, 346, 1055

Kauffmann, G., Heckman, T. M., White, S. D. M., et al. 2003b, MNRAS, 341, 33

Kewley, L. J., Groves, B., Kauffmann, G., \& Heckman, T. 2006, MNRAS, 372, 961

Mulchaey, J. S., Wilson, A. S., \& Tsvetanov, Z. 1996, ApJS, 102, 309

Nakamura, O., Fukugita, M., Yasuda, N., et al. 2003, AJ, 125, 1682

Oh, K., Sarzi, M., Schawinski, K., \& Yi, S. K. 2011, ApJS, 195, 13

Sandage, A., \& Tammann, G. A. 1981, in Carnegie Inst. of Washington, Publ., 635

Sarzi, M., Shields, J. C., Schawinski, K., et al. 2010, MNRAS, 402, 2187

Shen, S., Mo, H. J., White, S. D. M., et al. 2003, MNRAS, 343, 978

Stasińska, G., Vale Asari, N., Cid Fernandes, R., et al. 2008, MNRAS, 391, L29

Strauss, M. A., Weinberg, D. H., Lupton, R. H., et al. 2002, AJ, 124, 1810

Tremaine, S., Gebhardt, K., Bender, R., et al. 2002, ApJ, 574, 740

Trinchieri, G., \& di Serego Alighieri, S. 1991, AJ, 101, 1647

Veilleux, S., \& Osterbrock, D. E. 1987, ApJS, 63, 295

York, D. G., Adelman, J., Anderson, Jr., J. E., et al. 2000, AJ, 120, 1579 